\title{
BOUNDARY ELEMENT SOLUTION TO SIZE-DEPENDENT PIEZOELECTRIC BIMATERIALS
}

\author{
AREZOO HAJESFANDIARI \\ University at Buffalo, Buffalo \\ New York, 14226, USA \\ ah62@buffalo.edu
}

Key words: Integral Equations, Piezoelectricity, Flexoelectricity, Biomaterials, Micromechanics, Size-dependent Multiphysics

\begin{abstract}
A boundary element formulation based on the consistent couple stress theory is used to analyze two-dimensional size-dependent piezoelectric response in isotropic dielectric materials. In this approach, there exist a size-dependent piezoelectricity or flexoelectricity effect for centrosymmetric materials. The size-dependent effect is specified by one characteristic length scale parameter $l$, and the electromechanical effect is specified by one flexoelectric coefficient $f$. This phenomenon is a coupled problem involving mechanical and electrical effects. A boundary-only formulation is used for which the primary variables are displacements, rotations, force-tractions, couple-tractions, electric potential, and normal electric displacement. This BEM formulation is applied to a bimaterial computational problem to confirm the validity of the numerical implementation and to explore the physics of the flexoelectric coupling.
\end{abstract}

\section{INTRODUCTION}

The classical continuum mechanics predicts the piezoelectric effects only for noncentrosymmetric materials. But experiments show that such an effect can be anticipated when the material is centrosymmetric, such as isotropic or cubic materials. Unlike the classical theory, this effect cannot be explained by the coupling of the polarization and strain. To be able to understand the piezoelectric effect in centrosymmetric materials, a size-dependent continuum theory is needed. In the fully consistent flexoelecticity [1], these effects are related to the coupling of the polarization and mean curvature. This theory includes length scales and considers rotation as a primary degree of freedom [2]. The couple stress flexoelectricity involves only one length scale parameter and one flexoelectricity coefficient, which makes it very convenient.

\section{SIZE-DEPENDENT FLEXOELECTRICITY}

The governing equations for two-dimensional size-dependent flexoelectricity include the force balance, the moment balance, and the electric Gauss law as following [1]

$$
\sigma_{j i, j}+F_{i}=0
$$




$$
\begin{aligned}
\mu_{j i, j}+\varepsilon_{i j k} \sigma_{j k} & =0 \\
D_{i, i}+\rho_{E} & =0
\end{aligned}
$$

where $\sigma_{j i}$ is the force-stress tensor, $\mu_{j i}$ is the skew-symmetric couple-stress tensor, and $D_{i}$ the electric displacement vector. $F_{i}$ is the body force, $\rho_{E}$ is the electric charge density is the volume, and $\varepsilon_{i j k}$ is the three-dimensional alternating or Levi-Civita symbol. Note that the force-traction $t_{i}$, couple-traction $m_{i}$ and normal electric displacement $d$ on the surface of an element are defined as

$$
\begin{gathered}
t_{i}=\sigma_{j i} n_{j} \\
m_{i}=\mu_{j i} n_{j} \\
d=D_{i} n_{i}
\end{gathered}
$$

where $n_{i}$ is the normal unit vector on the surface. The constitutive relations for an isotropic dielectric material are

$$
\begin{gathered}
\sigma_{(j i)}=\lambda e_{k k} \delta_{i j}+2 \mu e_{i j} \\
\mu_{i}=-8 G l^{2} \kappa_{i}+2 f E_{i}
\end{gathered}
$$
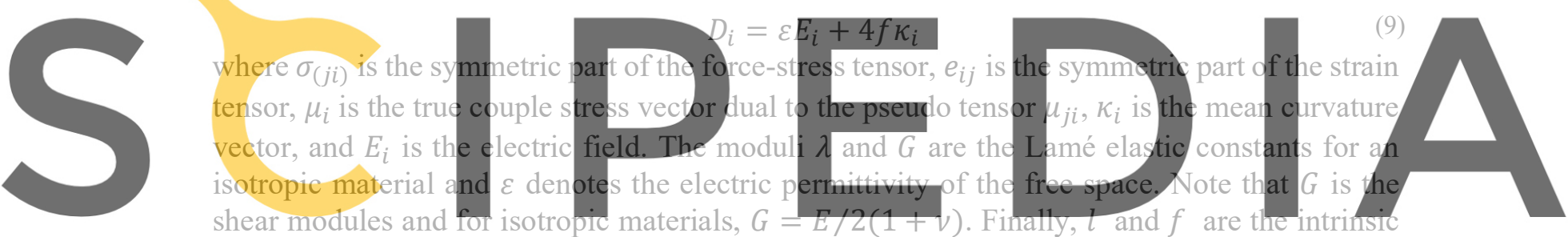

length scale parameter and the flexoelectric coefficient respectively. They are both properties

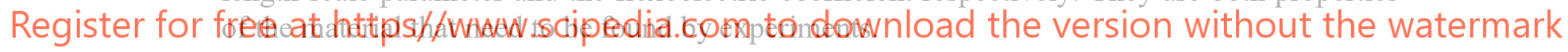

After using the constitutive relations (7)-(9), the governing equations (1)-(3) reduce to the mechanical and electrical equilibrium equations as following:

$$
\begin{gathered}
{\left[\lambda+G\left(1+l^{2} \nabla^{2}\right)\right] u_{k, k i}+G\left(1-l^{2} \nabla^{2}\right) \nabla^{2} u_{i}+F_{i}=0} \\
\varepsilon \nabla^{2} \varphi+\rho_{E}=0
\end{gathered}
$$

where $u_{i}$ is the displacement vector and $\varphi$ is the electric potential. Note that in the consistent size-dependent flexoelectricity, the three primary degrees of freedom are the displacement $u_{i}$, rotation $\omega_{i}$, and potential $\varphi$ such that

$$
\begin{gathered}
e_{i j}=\frac{1}{2}\left(u_{i, j}+u_{j, i}\right) \\
\kappa_{i}=\frac{1}{2} \varepsilon_{i j k} \omega_{k, j} \\
E_{i}=-\varphi_{, i}
\end{gathered}
$$


In order to solve equations (10)-(11) we need boundary conditions in the form of either essential or natural boundary conditions for each degree of freedom. Displacement, rotation and potential are the essential boundary conditions and the corresponding natural boundary conditions are force-traction $t_{i}$, couple-traction $m_{i}$, and normal electric displacement $d$.

\section{BOUNDARY ELEMENT IMPLEMENTATIONS}

Analytical solution is available to equations (10)-(11) for some planar size-dependent flexoelectric problems, but for a general application of the theory to model a wide range of problems, a robust numerical method is required. In the present work, boundary element method is used to solve a biomaterial flexoelectric problem with a simple geometry. The details on the integral representation of the formulation using the reciprocal theorem is provided in [3]. These integral representations are then discretized and implemented as a boundary element method formulation. It will be in the form of a set of algebraic linear equations.

To be able to use the formulation for multiregion problems, we need to write the integral equations for each region separately. Then, by enforcing appropriate interface conditions, we bring the two sets of equations together. It is noteworthy to mention that for perfectly bounded interfaces, the interface conditions are the continuity of displacements $u_{i}$ and rotations $\omega_{i}$, and equilibrium of force-traction $t_{i}$ and couple-tractions $m_{i}$ of the two regions.
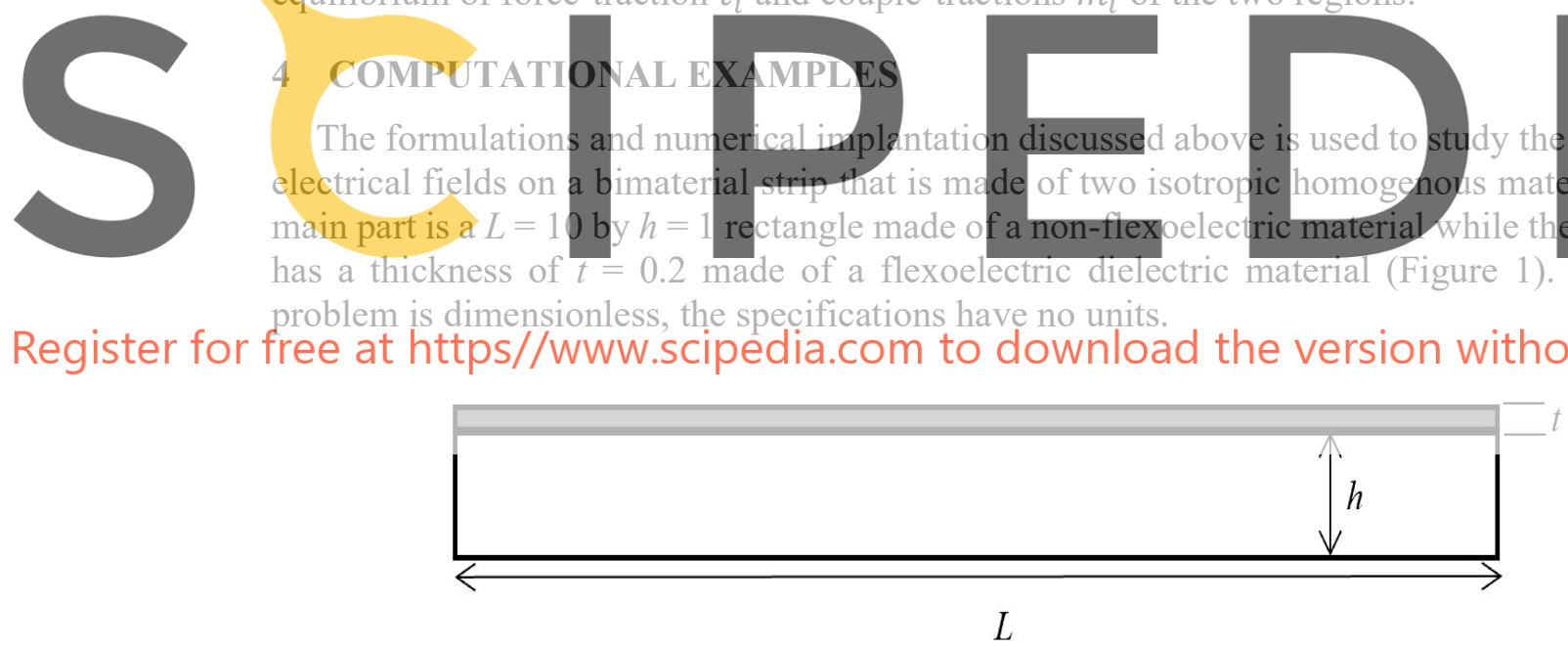

Figure 1: Bimaterial strip with a flexoelectric material on the top

The idea is to place this strip in an electric field and see how the flexoelectric top layer responds to the electrical field and makes the whole object deform. The properties of both materials are given in Table 1. Note that the couple-stress parameter is defined as $\eta=G l^{2}$. 
Table 1: Material properties

\begin{tabular}{ccc}
\hline & Rectangle & Top Layer \\
\hline Modules of Elasticity $(E)$ & 5.2 & 2.0 \\
\hline Poisson Ratio $(v)$ & 0.25 & 0.2 \\
\hline Permittivity $(\varepsilon)$ & 1.0 & 1.0 \\
\hline Flexoelectricity $(f)$ & 0. & -1.0 \\
\hline Couple-stress Parameter $(\eta)$ & 1.0 & 0.5 \\
\hline
\end{tabular}

\subsection{Example I: Free with potential difference}

As the first example, we place the strip in an electrical domain, such that the potential on the top and bottom surface are different. On the other hand, the two side surfaces are electrically insulated, i.e. $d=0$. The strip is free, but to prevent rigid body motion, the midpoint on the lower surface is fixed in the vertical direction, i.e. $u_{2}=0$ (Figure 2 top).
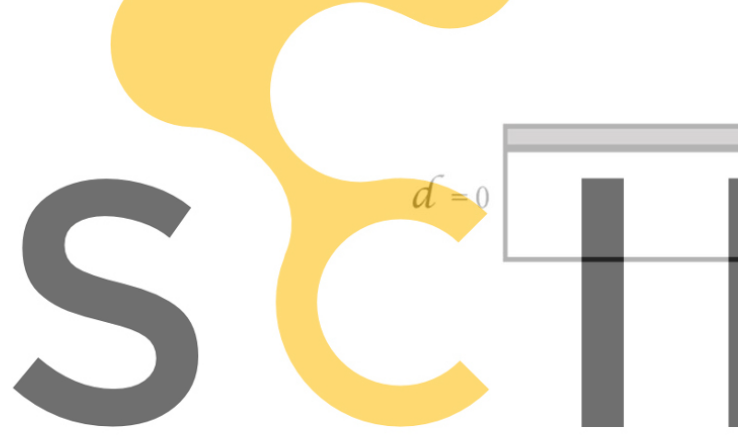

$$
\phi=0
$$

$$
u_{2}=0 \quad \phi=1
$$

Figure 2: Free strip with potential difference, full (top), half (bottom)

It is seen in the figure that the geometry of the problem and boundary conditions are symmetric with respect to the vertical midline. Thus, for simplicity, the analysis can be done for half of the strip, as shown in Figure 2 bottom. Boundary conditions for the midline are prescribed based on the symmetry of the problem. Thus, the horizontal displacement $u_{1}$ and rotation $\omega_{3}=\omega$ are zero.

As mentioned above, the top layer is made of a flexoelectric material. The difference in the electric potential at the top and bottom surfaces of the strip creates an electric field which leads to mechanical stresses in the flexoelectric material. Since the strip is free, the top layer bends due to those stresses and makes the entire strip bend.

The change in the normal force-tractions $t_{1}$ and $t_{2}$ and couple-tractions $m_{3}=m$ along the 
vertical midline and along the horizontal interface are presented in Figures 3 and 4, respectively.
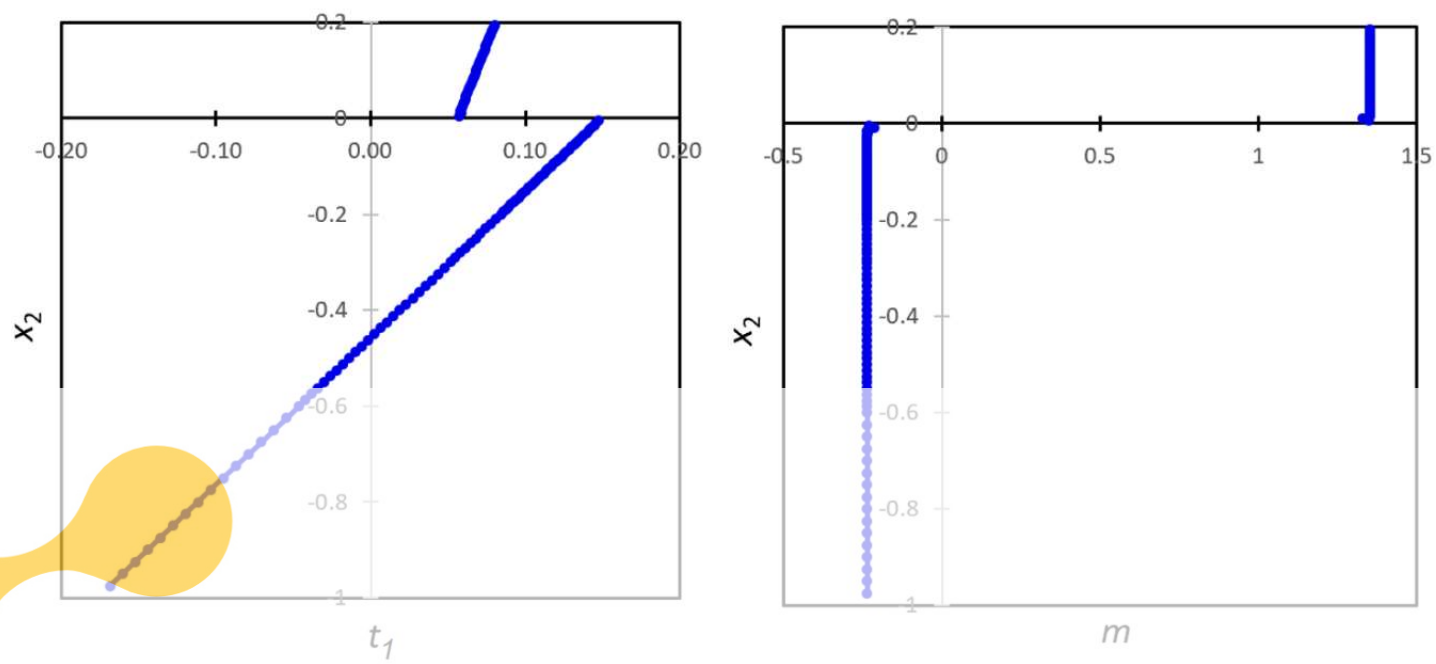

Figure 3: Normal force-traction $t_{l}$ and couple-traction $m$ along vertical midline for Example I
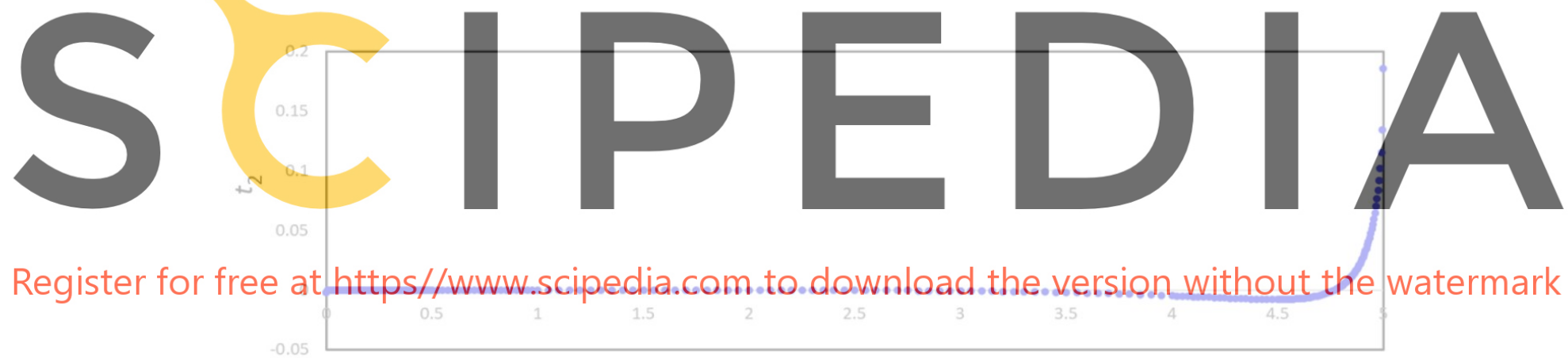

$E^{4}{ }^{4}$

$x_{1}$

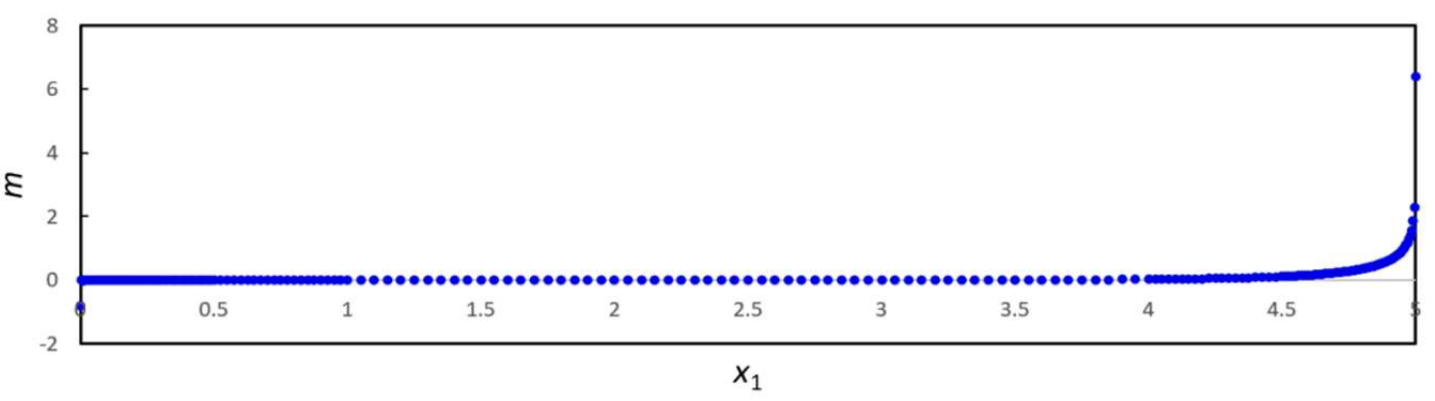

Figure 4: Normal force-traction $t_{2}$ and couple traction $m$ along the interface for Example I

\subsection{Example II: Bottom on rollers with potential difference}

In the next example, the strip is not free to bend anymore. The bottom surface is constrained in the vertical direction, while it is still free to rotate or move horizontally as shown in Figure 
5. Due to this constraint, force-tractions are created in the bottom surface. However, since this surface is free to rotate, the couple-traction is zero.

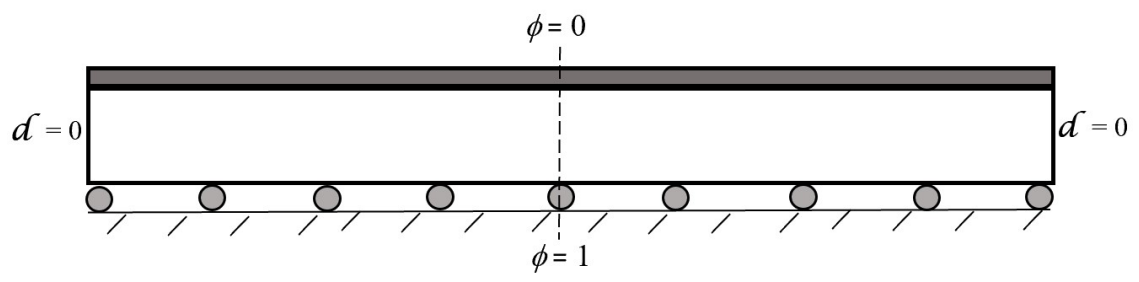

Figure 5: Bimaterial strip on rollers with potential difference

Apparently, the strip cannot bend, but there is some deformation in the top flexoelectric layer, specially near the corners of the interface area. This deformation can lead to delamination in the structure which makes the layer gradually start to separate from the base strip. This is a design issue which needs to be resolved for such structures.

Figure 6 represents the change in the normal force- and couple-tractions along the vertical midline, while in Figures 7 and 8, the normal force-traction along the bottom surface $t_{2}$, and the
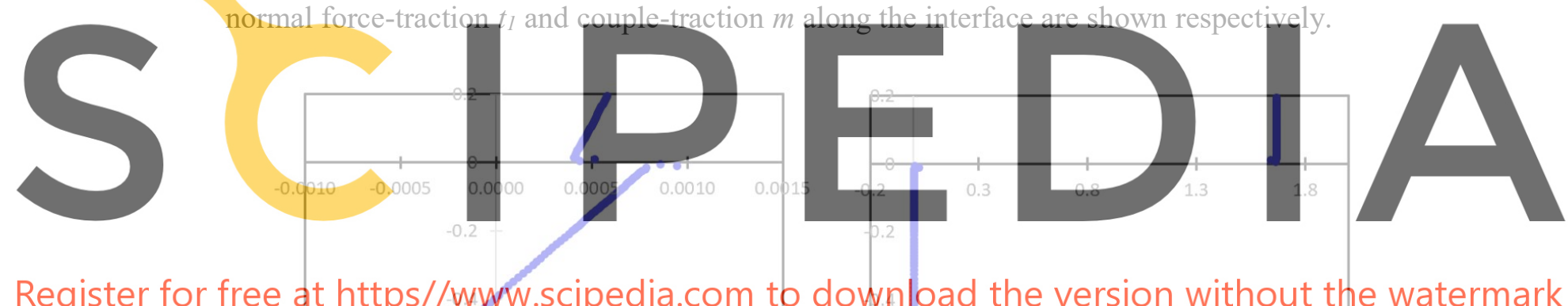

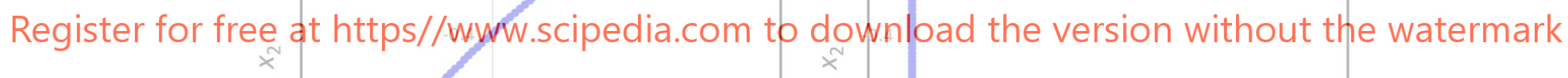
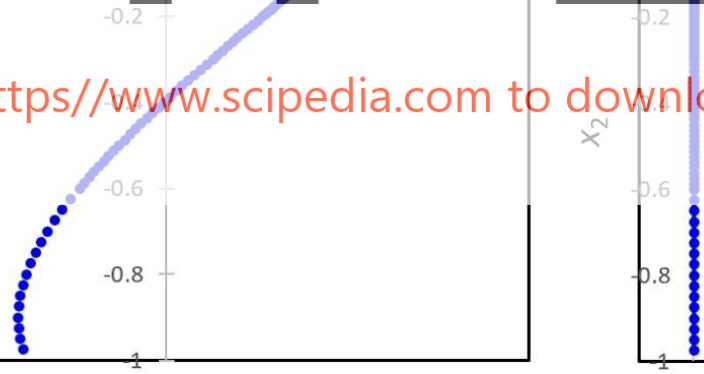

$t_{1}$

$m$

Figure 6: Normal force-traction $t_{1}$ and couple-traction $m$ along vertical midline for Example II 


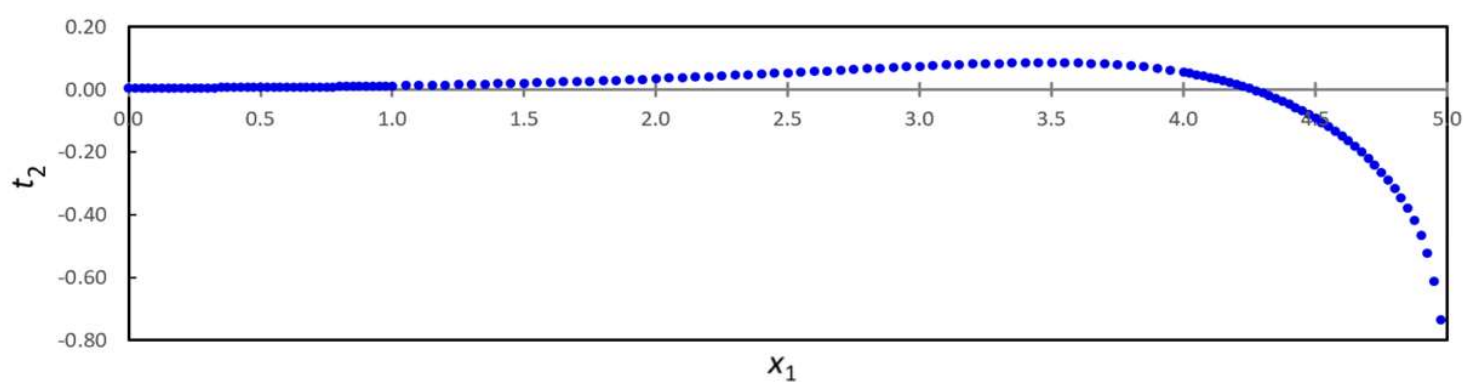

Figure 7: Normal force-traction $t_{2}$ along the bottom surface for Example II

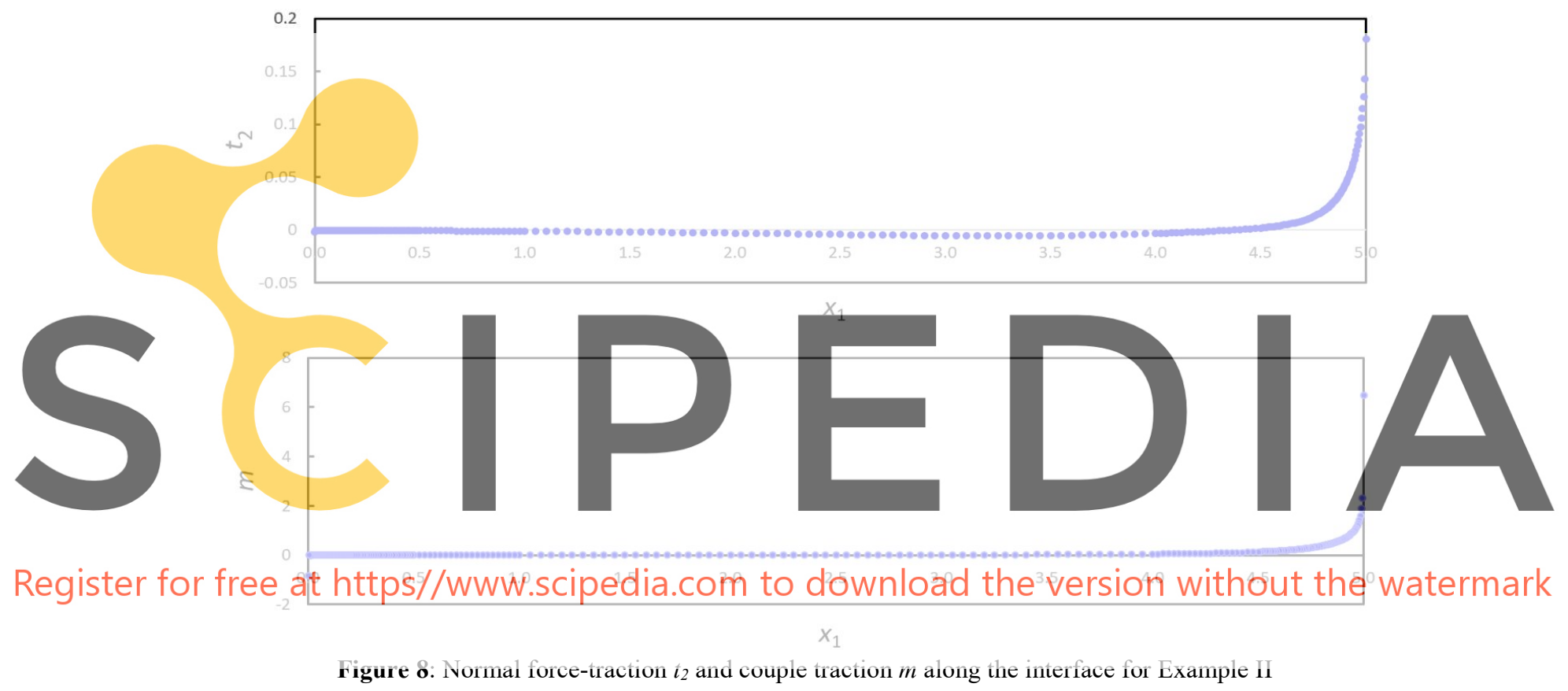

\subsection{Example III: Fully fixed bottom with potential difference}

The third example also involves the fixing of the bottom surface, but this time it is fully fixed. This means that the vertical and horizontal motions and the rotation are constrained on this surface (Figure 9). As a result of the electric field, couple-tractions will also be created on the bottom surface.

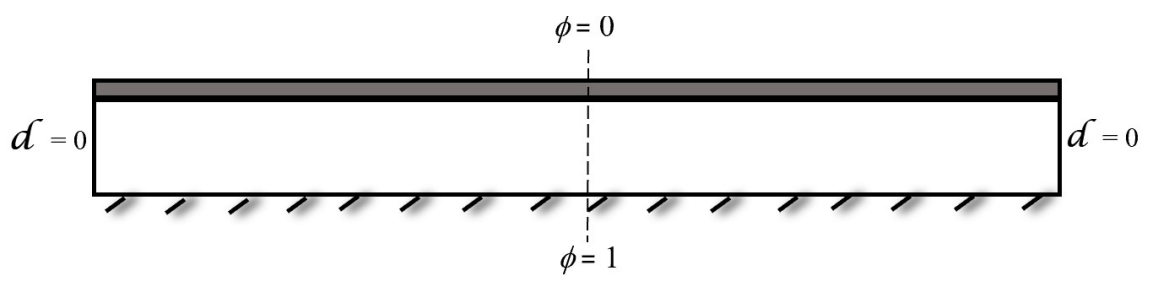

Figure 9: Bimaterial strip with fully fixed bottom surface and potential difference 
The normal force-tractions and couple-tractions are shown in Figures 10, 11, and 12 along the vertical midline, bottom surface, and the interface, respectively.
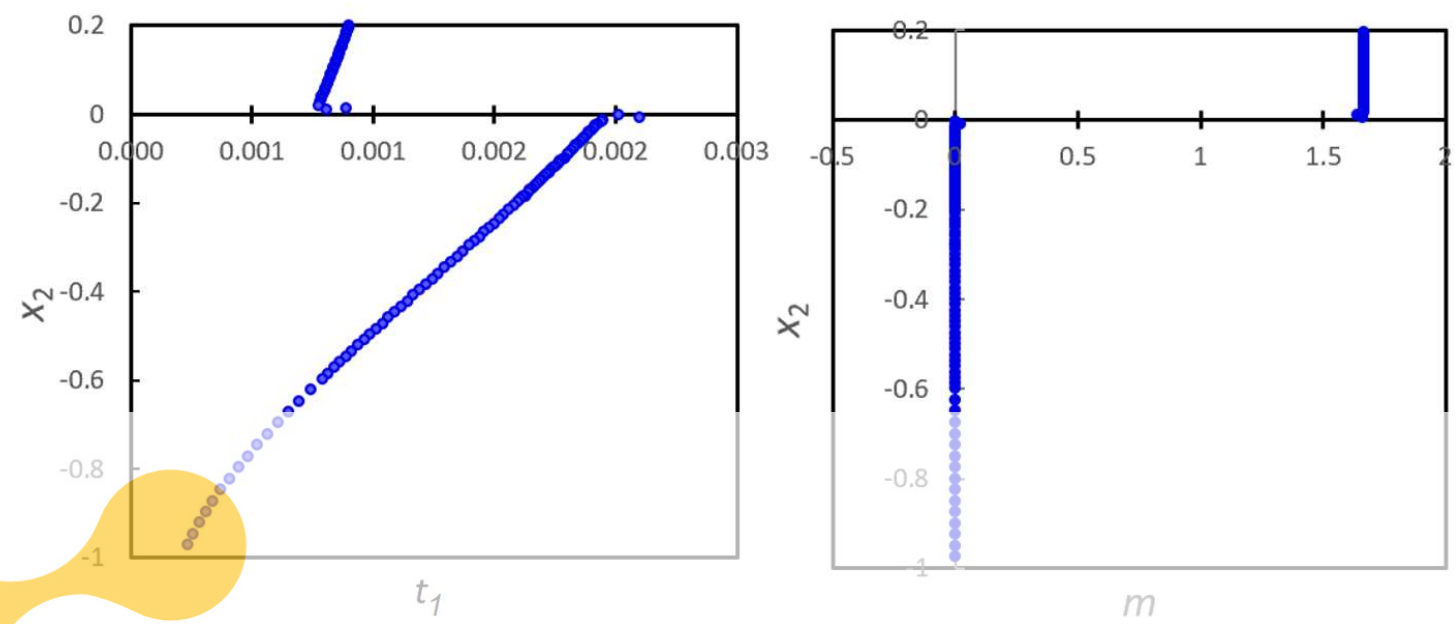

Figure 10: Normal force-traction $t_{1}$ and couple-traction $m$ along vertical midline for Example III
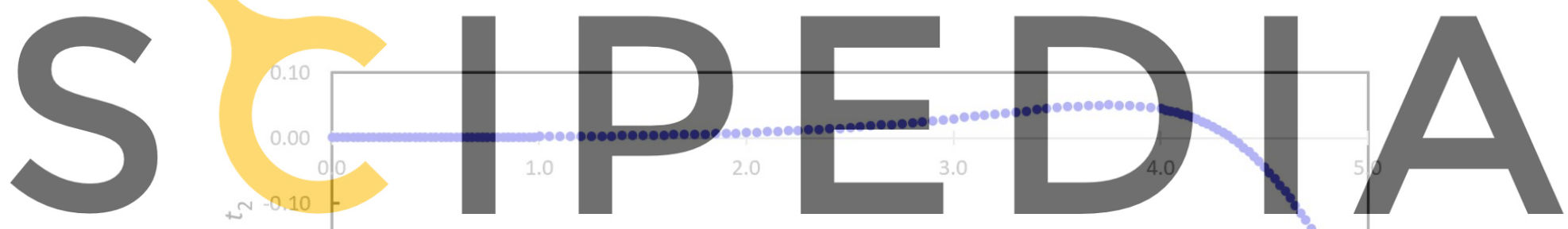

Register for free at https//www.scipedia.com to download the version without the watermark

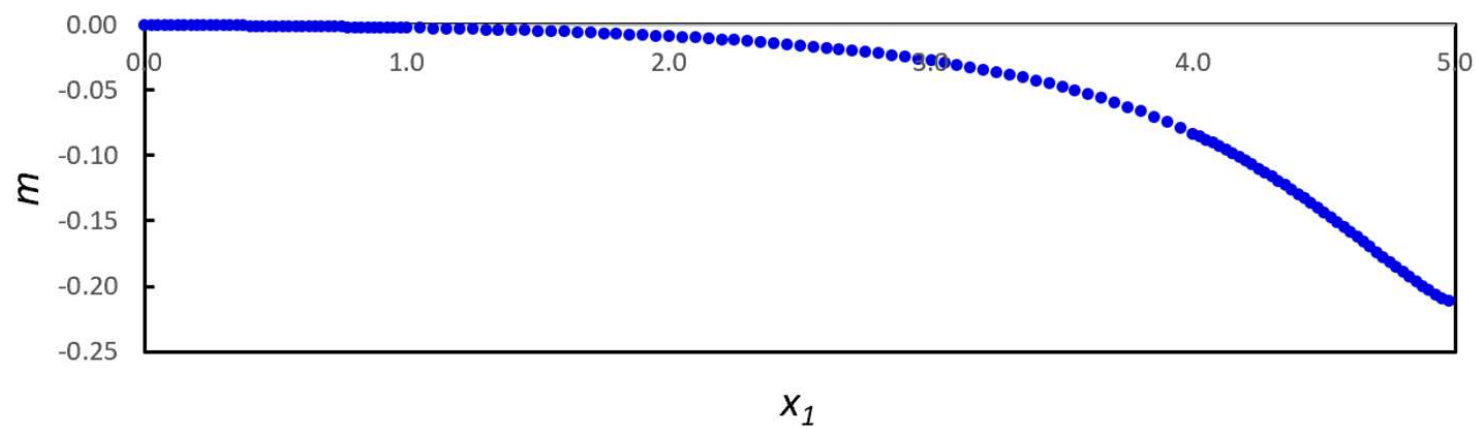

Figure 11: Normal force-traction $t_{2}$ and couple-traction $m$ along the bottom surface for Example III 

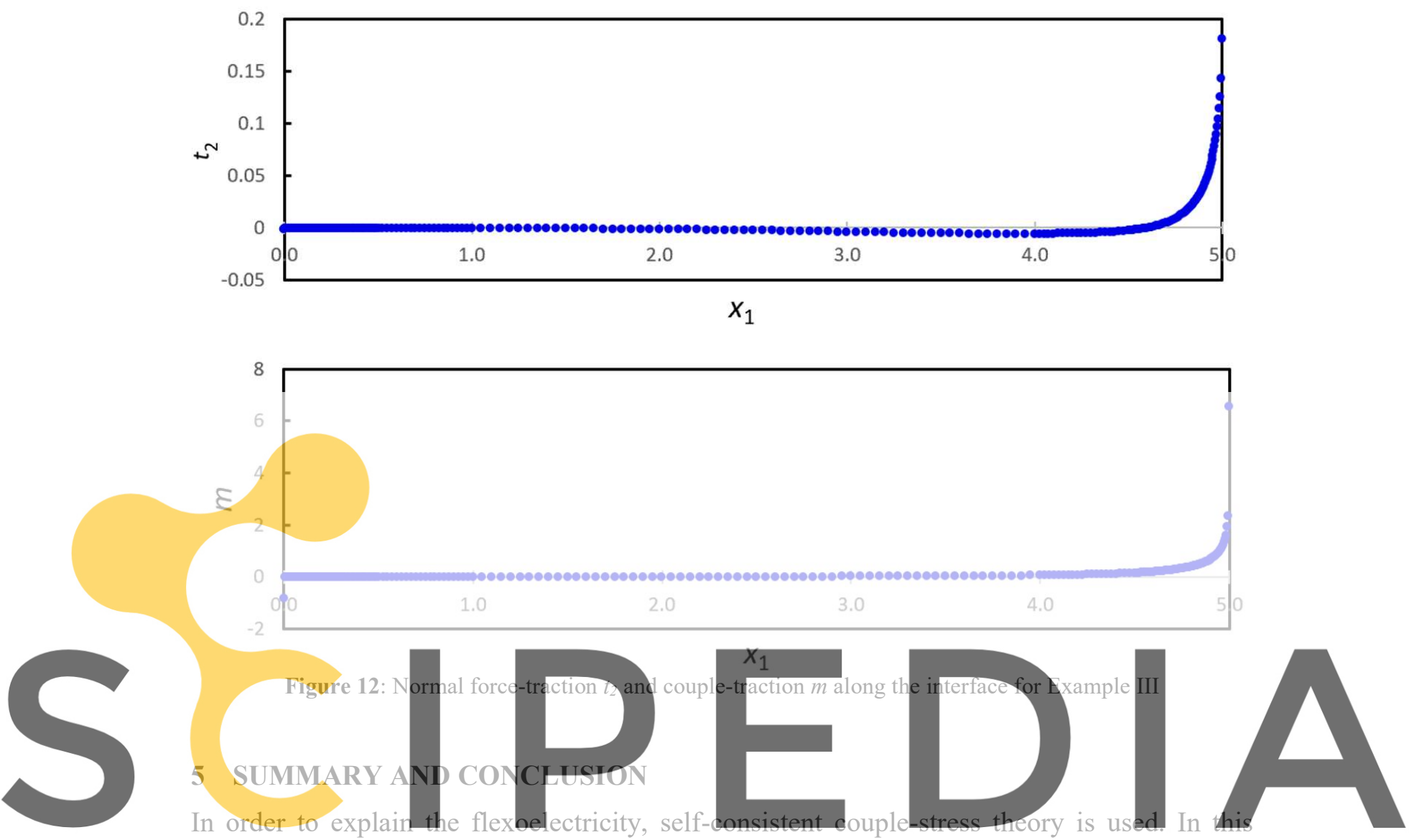

theory, rotation is a degree of freedom. The governing equations involve two coefficients only,

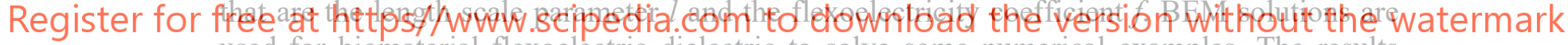
used for biomaterial flexoelectric dielectric to solve some numerical examples. The results show good convergence and predict delamination in the structure due to electric field.

\section{REFERENCES}

[1] Hadjesfandiari, A.R. Size-dependent piezoelectricity, Int. J. Solids Struct (2013) 50:27812791.

[2] Hadjesfandiari, A.H. and Dargush, A.H. Couple stress theory for solids. Int. J. Solids Struct (2011) 48:2496-2510.

[3] Hajesfandiari, A., Hadjesfandiari, A.R., and Dargush, G.F. Boundary element formulation for plane problems in size-dependent piezoelectricity. Int. J. Numer. Meth. Engng (2016) 108(7):667-694. 